\title{
A estrutura científica da inteligência competitiva'
}

\author{
The scientific structure of competitive intelligence
}

Elaine Coutinho MARCIAL²

Emir José SUAIDEN²

\section{Resumo}

A estrutura científica de uma área é a base para o seu reconhecimento como disciplina científica. Sendo a Inteligência Competitiva uma área recente do conhecimento, objetiva-se com esta pesquisa a proposição de sua estrutura científica e sistema de investigação nos níveis epistemológico, científico e aplicado. Busca-se também identificar o paradigma da Inteligência Competitiva, enumerar as teorias e modelos que a fundamentam e listar as soluções de problemas da vida real propostos por essa área. Trata-se de pesquisa descritiva cujo método utilizado foi o levantamento e análise estatística do comportamento de variáveis de um modelo sistêmico de definição de objeto científico em uma amostra formada por artigos científicos publicados em língua inglesa e portuguesa. Os resultados mostram a existência de estudo nos três níveis da estrutura científica. Há três paradigmas que regem a pesquisa nessa área. Apresenta corpo teórico que apoia estudos desse campo e que é utilizado para auxiliar na solução de problemas de ordem prática. Há pesquisas em nível aplicado, destacando-se as relacionadas à melhoria do processo de Inteligência Competitiva e à contribuição desta área a outros processos organizacionais. Conclui-se que a proposta explicitada de estrutura científica e de sistema de investigação, nos níveis epistemológico, científico e aplicado, para a Inteligência Competitiva é adequada à compreensão desta disciplina.

Palavras chave: Ciência da Informação. Estrutura científica. Inteligência competitiva. Paradigma.

\begin{abstract}
The scientific framework of a new field of knowledge is the foundation for its recognition as a scientific discipline. Considering that competitive intelligence is a recent field of knowledge, the main purpose of this study is to propose a scientific framework for competitive intelligence and a hierarchical research system with practical, scientific and epistemological perspectives levels. Other purposes include identifying the paradigm of competitive intelligence, enumerating its theories and models, and listing the solutions for real world problems proposed by this field. This is a descriptive research that uses the survey method and statistical analysis of variable behavior from a systemic model for defining scientific objects, using a sample of scientific articles published both in English and Portuguese. The results showed the existence of three scientific levels of research in this field. The following three paradigms support research in this field: a theoretical framework that supports studies in the field that are used to help solving practical problems; applied studies, particularly those related to improving the process of competitive intelligence; and those that contribute to other organizational processes. It may be concluded that the explicit scientific framework and a hierarchical research system proposed in the epistemological, scientific and practical levels are suitable for understanding this discipline.
\end{abstract}

Keywords: Information Science. Scientific structure. Competitive intelligence. Paradigm.

\section{Introdução}

Apesar da crescente evolução da Inteligência Competitiva (IC), tanto no campo da pesquisa quanto no da prática organizacional, verificou-se que a mesma carecia de fundamentação teórica organizada e explicitada, destacando sua estrutura científica de forma consistente e sistematizada. Havia também necessidade da

\footnotetext{
1 Artigo elaborado a partir da tese de doutorado de E.C. MARCIAL, intitulada "Aspectos fundamentais da inteligência competitiva e a Ciência da Informação". Universidade de Brasília, 2013

2 Universidade de Brasília, Faculdade de Ciência da Informação, Programa de Pós-Graduação em Ciência da Informação. Campus Universitário Darcy Ribeiro, 70910-900, Brasília, DF, Brasil. Correspondência para/Correspondence to: E.C. MARCIAL. E-mail:<elamarcial@gmail.com>.

Recebido em 25/3/2014, reapresentado em 13/1/2015 e aceito para publicação em 20/3/2015
} 
identificação de como essa estrutura científica era utilizada formalmente para apoiar a resolução dos seus problemas de pesquisa.

Comparado com a evolução da ciência, a Inteligência Competitiva é um campo recente. Surgiu como prática organizacional na década de 1950, na Europa e no Japão, e até a década de 1970 apresentou evolução mais relacionada à atuação profissional do que à pesquisa. Exemplo dessa fundamentação teórica pouco explícita foi citada na análise inicial realizada em quatro artigos do Journal of Competitive Intelligence and Management Dishman et al. (2003); Fleisher et al. (2003); Knip et al. (2003); e Fleisher et al. (2007). Esses autores apresentaram levantamento de publicações que abordavam o tema Competitive Intelligence, categorizadas em livros, monografias e artigos científicos, cobrindo o período de 1930 a 2006 e somente em língua inglesa.

Constatou-se, com a leitura e análise dos títulos desses documentos, que a primeira publicação que versava especificamente sobre Inteligência Competitiva foi publicada em 1959. Em mais de 3 mil citações levantadas pelos cinco autores foram encontradas somente quatro que trataram de questões fundamentais da IC: Prescott (1995, 1996, 1999) e Serpa (2000).

Nesse contexto, levantamentos e análises realizados por: (1) Mueller et al. (2000), abordando o surgimento de novos temas no âmbito do IV Encontro Nacional de Pesquisa em Ciência da Informação, citaram a IC como tema emergente; e (2) Marcial e Ornelas (2007) constataram, em relação às citações de publicações científicas sobre IC encontradas nos currículos Lattes de doutores no Brasil, que a maioria dos trabalhos versa sobre estudos de caso e nenhum sobre questões epistemológicas da IC.

Além dessas questões, constatou-se a falta de consenso sobre o que vem a ser Inteligência Competitiva. Diversas definições foram encontradas na literatura como as de Fuld (1995), Kahaner (1996), Tyson (1998), Prescott (1999), Miller (2000), Tarapanoff (2001) e Fleisher e Babette (2003). A área caracteriza-se como interdisciplinar, conforme defendido por Platt (1974), Taborda e Ferreira (2002) e Marcial e Ornelas (2007), e apresenta áreas do conhecimento correlatas: ciência da informação, administração, ciência da computação e psicologia. Essas evidências sinalizavam a falta de uma estrutura científica e sistema de investigação explícito para a IC que delimitassem sua fundamentação teórica.

\section{Filosofia da ciência}

A base desta investigação científica foi pautada no conceito de que uma disciplina científica e seu sistema de investigação poderiam ser representados por uma estrutura hierárquica constituída de três níveis: aplicado, científico e epistemológico, segundo a teoria de sistemas multiníveis apresentada por Gigch e Pipino (1986). As características de cada um desses níveis, que apresentam interrelações sistêmicas entre eles estão descritas a seguir:

Nível epistemológico: também chamado de conceitual, meta-nível ou meta-modelagem. Representa o quadro conceitual e metodológico de uma determinada comunidade científica. É a atividade de pesquisa que procura definir a origem do conhecimento da disciplina, justificar seus métodos de raciocínio e enunciar sua metodologia. Trata de questões filosóficas, epistemológicas e teóricas sobre a ciência. As investigações desse nível têm como produto a definição de um paradigma capaz de nortear essa ciência.

Nível científico: Também chamado de nível de modelagem ou do objeto. Representa o nível de desenvolvimento, formulação e validação de teorias, métodos e modelos. Os produtos são as teorias, métodos e modelos desenvolvidos sob a égide do paradigma definido no meta-nível. Seu propósito é desenvolver e ampliar o conhecimento que pode ser utilizado pelo sistema de investigação do nível aplicado, podendo tomar emprestado conhecimentos de outras disciplinas.

Nível aplicado: Também chamado de nível empírico, de aplicação ou inferior de investigação. Representa o nível de solução de problemas da vida real para os quais se aplicam as teorias, modelos, técnicas e tecnologias idealizadas nos outros níveis. Caracteriza-se pela utilização de métodos de investigação empíricos, como os estudos de caso, os estudos de campo, os testes de campo e os estudos laboratoriais. Seu produto é a solução de problemas organizacionais.

Essa abordagem sistêmica defende a importância do paradigma para uma ciência. Conforme abordado por Kuhn (2006), os empreendimentos científicos são nor- 
teados por paradigmas e a ciência evolui basicamente de duas formas: (1) acumulativa, por meio da pesquisa normal, ao solucionar quebra-cabeças, tendo como base um paradigma que orienta esse processo; e (2) por meio de revoluções científicas, quando o paradigma vigente é contestado, pois não é capaz de solucionar as anomalias emergentes. A crise instaurada na ciência normal somente seria solucionada com o surgimento de um novo paradigma aceito pela comunidade científica que substitui ou complementa o anterior (Kuhn, 2006).

Independentemente da forma como a ciência evolui a existência de um paradigma que norteie sua evolução é fundamental. Eles indicam as crenças, valores e técnicas partilhadas por uma comunidade científica ao mesmo tempo que fornecem, aos fenômenos observados pelos cientistas, soluções concretas de quebra-cabeças. São fontes de teorias, métodos, modelos e padrões de solução aceitos por qualquer comunidade científica amadurecida (Kuhn, 2006).

Nesse contexto, o objetivo desta pesquisa foi propor uma estrutura científica e um sistema de investigação nos níveis epistemológico, científico e aplicado da Inteligência Competitiva. Buscou-se, também, identificar o paradigma da IC e estabelecer sua fundamentação epistemológica, enumerar as teorias e modelos que embasam e estabelecem sua fundamentação científica, apontar as soluções de problemas da vida real propostas pela IC e estabelecer sua fundamentação aplicada. Com esses objetivos buscou-se responder a seguinte questão: qual a estrutura científica e o sistema de investigação nos níveis epistemológico, científico e aplicado da Inteligência Competitiva? Assim, o estudo, elaborado no âmbito da Ciência da Informação, justificou-se no fato de a IC ter como possível objeto de pesquisa a informação no processo de tomada de decisão, considerando inclusive as suas fases de produção (Prescott, 1995).

\section{Procedimentos metodológicos}

A pesquisa teve duração de quatro anos (2010 a 2013) e pode ser classificada como descritiva. O método utilizado foi o bibliográfico estruturado sob a abordagem sistêmica para a compreensão do objeto científico nos níveis de investigação epistemológico, científico e aplicado, proposto por Gigch e Pipino (1986) e baseado na abordagem de Kuhn (2006). Foi realizado um levantamento estatístico do comportamento de variáveis em uma amostra da população e aplicados métodos estatísticos descritivos aos dados quanti e qualitativos obtidos.

Parte da metodologia apresentada a seguir seguiu os mesmos passos descritos por Marcial e Suaiden (2014) no que diz respeito à definição da população e da amostra utilizadas. A população foi formada por documentos, caracterizados como artigos científicos (publicados em periódicos nacionais e internacionais), teses e dissertações nacionais. As bases de dados pesquisadas também foram as mesmas utilizadas por Marcial e Suaiden (2014), sendo que foram realizadas buscas utilizando-se os termos "Inteligência Competitiva" e "Competitive Intelligence", para a identificação da lista de estratos amostrais que publicam artigos sobre IC - descrita em Marcial (2013). Foi considerada como uma restrição para esta pesquisa a população ter sido composta por publicações somente nas línguas inglesa e portuguesa. Estudos em áreas correlatas os quais não tinham como foco solucionar problemas específicos da IC, apesar de contribuir com ela, também não fizeram parte do levantamento.

Depurações foram realizadas, conforme Marcial e Suaiden (2014), e chegou-se a 125 unidades amostrais, totalizando 941 referências. Foi feita amostragem aleatória simples, estratificada proporcionalmente por estrato populacional. Os periódicos científicos e as bases de teses e dissertações brasileiras selecionadas foram definidos como estratos da população, por apresentarem características homogêneas em relação ao objetivo da pesquisa. A amostra foi estabelecida conforme fórmula de cálculo definida por Gil (1991) e descrito por Marcial e Suaiden (2014). A escolha dos artigos foi feita de forma aleatória, utilizando-se a função do Microsoft Excel 2010 de geração de números aleatórios (=ALEATÓRIO()). Os resultados foram multiplicados por 100, considerando apenas sua parte inteira.

No total foram analisados 315 documentos. A coleta e a classificação do conteúdo de cada variável foram realizadas manualmente pelo autor por meio da leitura de todos os documentos pertencentes à amostra selecionada. Os dados foram armazenados em um aplicativo desenvolvido em Microsoft Access para esse fim (Marcial \& Suaiden, 2014). Utilizou-se o software Statistical Package for the Social Sciences (IBM-SPSS) para a geração 
das estatísticas descritivas. As variáveis utilizadas para testar os pressupostos da pesquisa foram:

$v_{1}$ - Classificação da Pesquisa: a pesquisa pode ser classificada em três níveis: (1) epistemológico - se o objeto de pesquisa for a origem do conhecimento da disciplina, tratando de questões filosóficas, epistemológicas, ontológicas, éticas ou sobre princípios -, (2) científico - se o objeto for a formulação ou validação de teorias e modelos que são utilizados para descrever, explicar e prever o comportamento da disciplina e como ela se desenvolve -, (3) aplicado - se o objeto for a busca pela solução de problemas da vida real.

$v_{p}$ - Paradigma (produto do Nível Epistemológico): descrição do paradigma que rege a pesquisa. Utilizaram-se como possíveis paradigmas da IC aqueles propostos por Capurro (1991) para a Ciênica da Informação em função da aderência existente entre as duas áreas. São eles: (1) físico - para pesquisas centradas na teoria de recuperação da informação registrada, algo tangível como documentos, físicos ou eletrônicos, e informações armazenadas ou não em bases de dados-, (2) cognitivo: relacionado aos processos e modelos de produção da informação, conhecimento ou inteligência para atender às necessidades do demandante, bem como mapas cognitivos, modelos mentais ou de mundo -, (3) social - relacionado aos processos sociais de obtenção, produção e difusão da informação/Inteligência, bem como a utilização de redes humanas/sociais para apoiar tais processos.

$v_{t m}$ - Teorias, métodos e modelos da IC (produto do Nível Científico): lista de teorias, métodos, modelos e técnicas citados no documento.

$v_{s}$ - Soluções para problemas organizacionais (produto do Nível Aplicado): definição das soluções encontradas no nível aplicado.
$v_{a}$-Área de conhecimento do documento: classifica quanto a área de conhecimento onde foi realizada a pesquisa observando-se os conteúdos: filiação, curso e departamento dos autores, e área do periódico onde o documento foi publicado.

\section{Resultados e Discussão}

A população do presente estudo foi formada por 941 documentos científicos que apresentavam pesquisa exclusiva no âmbito da Inteligência Competitiva. A maior parte era de artigos científicos (74,9\%), seguidos por número significativo de dissertações $(21,0 \%)$. As teses representaram 4,1\% da população. Destaca-se que a maior parte das referências era em língua inglesa (68,3\%). Os artigos pertenciam a 125 periódicos que publicam a produção científica específica da área de Inteligência Competitiva, cuja lista pode ser encontrada em Marcial (2013). Esse volume sugeriu o quão dinâmica era a pesquisa nesse novo campo do conhecimento.

Da mesma forma que na pesquisa de Marcial e Suaiden (2014) a amostra foi formada por 315 documentos (33,5\% da população) de três tipos: artigos científicos (79,4\%), dissertações $(17,1 \%)$ e teses $(3,5 \%)$. O caráter interdisciplinar do sistema de investigação da IC é confirmado pelo perfil das unidades amostrais pertencentes a diversos campos do conhecimento, sendo que a Administração (43,2\%), a Ciência da Informação (22,4\%) e a própria área de Inteligência (11,2\%) foram aquelas que mais realizaram pesquisa no campo da IC.

A análise da estrutura hierárquica mostrou que existia uma estrutura científica da IC com produção nos três níveis: epistemológico, científico e aplicado (Tabela 1). A maior parte da pesquisa na área ocorria em nível de

Tabela 1. Nível da pesquisa no tempo.

\begin{tabular}{|c|c|c|c|c|c|c|c|c|c|}
\hline \multirow{2}{*}{ Níveis } & \multicolumn{7}{|c|}{ Período } & \multirow{2}{*}{ Total } & \multirow{2}{*}{$\%$} \\
\hline & 1940 & 1950 & 1960 & 1970 & 1980 & 1990 & 2000 & & \\
\hline Contribuição Epistemológica & 0 & 0 & 1 & 1 & 0 & 10 & 32 & 44 & 14,0 \\
\hline Contribuiç̧ão Científica & 1 & 1 & 2 & 6 & 6 & 51 & 111 & 178 & 56,5 \\
\hline Contribuição Aplicada & 0 & 0 & 0 & 0 & 2 & 20 & 71 & 93 & 29,5 \\
\hline Total & 1 & 1 & 3 & 7 & 8 & 81 & 214 & 315 & 100,0 \\
\hline
\end{tabular}

Fonte: Elaborada pelos autores (2012-2013). 
contribuição científica (56,5\%), mas também havia significativa produção no nível aplicado (29,5\%).

Verificou-se que a pesquisa no nível científico existia desde a década de 1940 e foi uma constante em todo o período analisado (Tabela 1). Esse comportamento justifica-se pela necessidade do desenvolvimento de teorias, métodos e modelos para essa nova área.

Os estudos no nível epistemológico iniciaram-se na década de 1960, quando o conceito de IC entrou em debate. Essas pesquisas se estenderam durante os anos seguintes, tanto por se tratar de uma área nova, quanto pelo debate das questões éticas vinculadas à temática, conforme defendido por Prescott (1999). No nível aplicado, sua evolução iniciou na década de 1980, com o surgimento da necessidade de construção de um business case, também defendido por Prescott (1999). Esses dados sugerem que há uma evolução da Inteligência Competitiva como área de pesquisa.

A Tabela 2 apresenta a relação entre a classificação da pesquisa quanto ao nível na estrutura hierárquica e o método de pesquisa utilizado. Também mostra parte do sistema de investigação utilizado para IC, visto que, segundo Popper (2008), toda disciplina científica era formada por questões as quais eram respondidas com a utilização de métodos de pesquisa.

Havia diversidade de métodos de pesquisa utilizados (Tabela 2), sendo que a maioria utilizava-se de levantamento bibliográfico (41,0\%), estudo de caso $(26,0 \%)$ e levantamento estatístico (24,8\%). A totalidade das pesquisas que aplicavam o método histórico estava classificada no nível epistemológico. Já a maioria dos estudos de caso estava no nível aplicado e os levantamentos estatísticos no científico, o que confirma a natureza de cada um desses níveis.

O nível epistemológico foi representado pelas pesquisas realizadas no campo da filosofia da ciência e apresentou como principal produto o paradigma que norteia a ciência. Foram identificados três no campo da IC, sendo os mesmos observados por Capurro (1999) para a Ciência da Informação.

O paradigma predominante, que rege a pesquisa em Inteligência Competitiva, era o cognitivo (45,4\%) relacionado aos processos intelectuais de produção da informação/conhecimento/Inteligência, sendo também o mais antigo (década de 1940). Sua força estava ligada ao fato de a IC ser uma área voltada para a produção de informação estratégica, o que exige uso do intelecto na análise e interpretação dos dados e informações. Logo, pesquisas para aprimorar o processo de produção da Inteligência sempre tiveram espaço.

O paradigma físico era o segundo com maior ocorrência (37,1\%). Estava associado ao processo de reunião de dados e informação e ao seu tratamento automático, sendo também antigo (década de 1950). Isso porque, inicialmente, a atividade estava pautada na coleta de dados. $O$ crescimento da pesquisa regida por esse paradigma nas décadas de 1990 e 2000 poderia estar vinculado ao avanço das tecnologias da informação e comunicação durante o período e seu vínculo com a atividade de IC.

Tabela 2. Níveis de investigação e métodos de pesquisa.

\begin{tabular}{|c|c|c|c|c|c|}
\hline \multirow{2}{*}{ Método de pesquisa } & \multicolumn{3}{|c|}{ Classificação } & \multirow{2}{*}{ Total } & \multirow{2}{*}{$\%$} \\
\hline & Contribuição epistemológica & Contribuição científica & Contribuição prática & & \\
\hline Levantamento bibliográfico & 31 & 88 & 10 & 129 & 41,0 \\
\hline Estudo de caso & 1 & 2 & 79 & 82 & 26,0 \\
\hline Levantamento estatístico & 1 & 73 & 4 & 78 & 24,8 \\
\hline Método histórico & 10 & 0 & 0 & 10 & 3,2 \\
\hline Método comparativo & 0 & 4 & 0 & 4 & 1,3 \\
\hline Levantamento documental & 0 & 3 & 0 & 3 & 1,0 \\
\hline Pesquisa-ação & 0 & 2 & 0 & 2 & 0,6 \\
\hline Outros & 1 & 6 & 0 & 7 & 2,1 \\
\hline Total & 44 & 178 & 93 & 315 & 100,0 \\
\hline
\end{tabular}

Fonte: Elaborada pelos autores (2012-2013). 
O paradigma social (17,5\% das pesquisas) era recente (década de 1990) e emerge de características intrínsecas da IC - atividade humana pautada nas relações sociais. Destacou-se a gestão de redes sociais como um possível agente que facilitou a entrada e manutenção do profissional de Inteligência Competitiva no fluxo informacional, aumentando as chances de identificação de sinais fracos e possíveis mudanças ambientais futuras, conforme abordado por Mendes et al. (2010).

Já o nível científico estava representado pelas pesquisas cujo objetivo era desenvolver e expandir o conhecimento a ser utilizado na prática e passou a ocorrer com maior frequência a partir da década de 1990, com crescimento significativo na década de 2000. Os principais produtos eram as teorias, os métodos e os modelos para utilização aplicada. Foram encontradas 382 citações de categorias para a temática que embasavam a pesquisa no campo da IC ou eram propostas por estudos desenvolvidos nesse campo. Foram encontradas 1151 citações nos documentos analisados, sendo as categorias mais recorrentes: modelo (42,8\%), método (34,3\%) e teoria (12,9\%). Apenas em um percentual pequeno dos documentos analisados (4,3\%) não foi encontrada menção de nenhuma dessas categorias. Proporcionalmente, as teorias eram mais citadas nas teses e os métodos nas dissertações.

Dentre as teorias, métodos e modelos citados nas pesquisas desenvolvidas no âmbito da IC, o mais mencionado foi o modelo de ciclo de produção de Inteligência Competitiva (11,8\%), sendo ele o único desde a década de 1940. Os modelos de monitoramento ambiental (4,5\%) vêm logo em seguida. Apesar de terem sua origem na década de 1960 com as pesquisas de Aguilar (1967) e de estarem intimamente ligados à atividade de Inteligência, eles somente apareceram nas pesquisas sobre IC a partir da década de 1980. O método de análise da indústria de Porter (1992) foi o terceiro mais citado (4,3\%). Desenvolvido durante a década de 1980, foi incorporado à pesquisa no âmbito da Inteligência Competitiva a partir da década de 1990. Os métodos de reunião de informação e de difusão de IC, que fizeram parte do ciclo de produção de IC, ganharam pesquisas específicas a partir da década de 1950, classificando-se como o segundo mais antigo.
Dentre os métodos e modelos desenvolvidos especificamente para área de Inteligência Competitiva, os mais citados foram: o do ciclo de produção de IC, O modelo de Contrainteligência, o Key Intelligence Topics (KIT) e o método de early warning analysis. Os demais foram apropriados de outras áreas do conhecimento, como por exemplo, da Administração e da Ciência da Informação.

Os métodos de reunião e difusão de informação, etapas integrantes do ciclo de produção de IC, apareceram na década de 1950. Já as pesquisas que citavam o modelo de Inteligência Econômica surgiram nos anos 1960, assim como aquelas que associavam a Teoria da Comunicação à pesquisa na área de IC. Quanto às pesquisas que incluíam a preocupação em modelar os sistemas de IC, estas surgiram na década de 1970, de acordo com Prescott (1999). Os anos 1980 foram marcados pelo início da vinculação dos modelos de monitoramento ambiental e dos métodos de análise e construção de cenários à pesquisa no campo da IC. Também se iniciou a proposição de modelos de implantação de IC nas organizações.

Já o crescimento da pesquisa na área de IC foi a principal característica da década de 1990. Houve o crescimento de métodos e modelos que apoiavam a análise de IC, como os de Porter, de análise Strengths, Weaknesses, Opportunities, and Threats (SWOT), do uso da bibliometria e da preocupação com a pesquisa na área dos modelos de fluxo informacional. Também foi destaque desse período o início do aparecimento de citações sobre a Teoria Geral de Inteligência e de métodos e modelos específicos da área de IC, como os modelos de Contrainteligência, do KIT, de early warning e de blindspot. Já nos anos 2000, houve a vinculação da IC com a Teoria Geral da Administração. Também apareceram as primeiras pesquisas que citavam a técnica de entrevista como ferramenta de Inteligência Competitiva.

Foram identificas 77 teorias citadas ou propostas nos documentos analisados. As mais comuns são a Teoria Geral da Administração (16 documentos), a Teoria Geral de Inteligência (10 documentos) e a Teoria da Decisão (sete documentos). Se adicionarmos a frequência de ocorrência da Teoria Geral de Inteligência com a da Teoria da Inteligência (Intelligence Theory), a mesma passa para 13 documentos. A Teoria do Conhecimento também 
merece destaque. Apesar de representar a fundamentação do método de produção de Inteligência e da concepção teórica inicial de toda a atividade, conforme descrito por Platt (1974), ela foi pouco citada (quatro documentos).

Análise realizada, baseada no cruzamento das teorias, métodos e modelos mais citados com a origem da pesquisa, mostrou que o modelo de ciclo de produção de IC foi encontrado em documentos produzidos por todas as áreas de conhecimento que pesquisam Inteligência Competitiva (Tabela 3). Os modelos de monitoramento ambiental apareceram na maioria das áreas e foram o segundo mais citado, seguidos pelo método de análise da indústria de Porter. Esses resultados também confirmaram o caráter interdisciplinar da estrutura científica da IC.

No nível aplicado foram classificadas as pesquisas que apresentaram soluções para problemas organizacionais, utilizando-se de modelos e conhecimento desenvolvidos em nível científico. Nesse contexto, as contribuições dos estudos foram analisadas e divididas em duas categorias: (1) quanto à contribuição às demais áreas do conhecimento ou atividades realizadas pelas organizações (18,1\%); (2) melhorias do processo de IC (81,9\%) (Tabela 4).
A maior parte das pesquisas focou as contribuições para melhoria do processo de IC. Nessa categoria, o destaque foi o campo dos Sistemas de Inteligência Competitiva (SIC), representando 8,9\% das pesquisas. Havia estudos significativos que contribuíam para o entendimento da evolução da IC (6,0\%) e para a melhoria do processo de reunião da informação e monitoramento (4,1\% e 3,8\% das pesquisas, respectivamente) (Tabela 4). As principais contribuições da pesquisa em IC às demais áreas do conhecimento ou atividades realizadas pelas organizações estavam no campo do processo decisório, da gestão estratégica e dos resultados que a IC trazia para as organizações (Tabela 4).

Por fim, o Quadro 1 mostra, em síntese, os resultados do estudo no formato de uma proposta de estrutura científica e sistema de investigação para IC, com base no resultado do levantamento realizado.

\section{Conclusão}

A presente pesquisa teve como principal objetivo propor uma estrutura científica e um sistema de investigação nos níveis epistemológico, científico e aplicado para a Inteligência Competitiva. Para tanto, foi realizado levantamento de dados, obedecendo a abordagem

Tabela 3. Teorias, métodos e modelos mais citados e as área de conhecimento.

\begin{tabular}{|c|c|c|c|c|c|c|c|c|c|}
\hline Teorias, métodos e modelos & 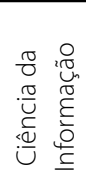 & 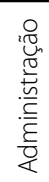 & 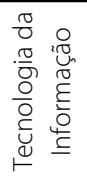 & 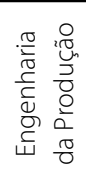 & 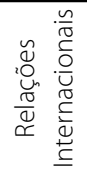 & 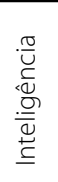 & 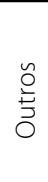 & Total & $\begin{array}{c}\text { Total } 315 \\
\text { artigos (\%) }\end{array}$ \\
\hline Modelo de ciclo de IC & 34 & 47 & 2 & 10 & 1 & 28 & 14 & 136 & 43,2 \\
\hline Modelo de monitoramento ambiental & 13 & 23 & 0 & 4 & 0 & 7 & 5 & 52 & 16,5 \\
\hline Método de análise da indústria - 5 forças de Porter & 10 & 24 & 1 & 6 & 0 & 4 & 4 & 49 & 15,6 \\
\hline Modelo de análise da matriz SWOT & 12 & 14 & 1 & 6 & 0 & 4 & 2 & 39 & 12,4 \\
\hline Método de análise e construção de cenários & 8 & 15 & 0 & 1 & 0 & 7 & 3 & 34 & 10,8 \\
\hline Método de benchmarking & 7 & 10 & 0 & 4 & 0 & 4 & 1 & 26 & 8,3 \\
\hline Método de análise dos Fatores Críticos de Sucesso & 3 & 9 & 0 & 6 & 0 & 2 & 2 & 22 & 7,0 \\
\hline Modelo de Contrainteligência & 5 & 7 & 0 & 1 & 1 & 3 & 2 & 19 & 6,0 \\
\hline Método de network analyze & 3 & 8 & 1 & 0 & 0 & 6 & 1 & 19 & 6,0 \\
\hline $\mathrm{KIT}$ & 2 & 7 & 0 & 1 & 0 & 6 & 2 & 18 & 5,7 \\
\hline Método de análise da concorrência de Porter & 6 & 8 & 0 & 0 & 0 & 3 & 0 & 17 & 5,4 \\
\hline Teoria Geral da Administração & 3 & 6 & 0 & 3 & 0 & 1 & 3 & 16 & 5,1 \\
\hline Método de early warning analysis & 2 & 5 & 0 & 0 & 0 & 6 & 1 & 14 & 4,4 \\
\hline Método de text mining & 3 & 2 & 3 & 1 & 0 & 0 & 5 & 14 & 4,4 \\
\hline
\end{tabular}

Fonte: Elaborada pelos autores (2012-2013).

Nota: IC: Inteligência Competitiva; KIT: Key Intelligence Topics; SWOT: Strengths, Weaknesses, Opportunities and Threats. 
Tabela 4. Contribuição prática da pesquisa no campo da Inteligência Competitiva.

\begin{tabular}{|c|c|c|c|}
\hline Contribuição prática da pesquisa no campo da IC & fi & $\%$ & Categoria (\%) \\
\hline Contribuição da IC & 57 & 18,1 & 100,0 \\
\hline Contribuição da IC ao processo decisório & 14 & 4,4 & 24,6 \\
\hline Contribuição da IC na gestão estratégica & 12 & 3,8 & 21,1 \\
\hline Contribuição da IC para resultado organizacional & 8 & 2,5 & 14,0 \\
\hline Contribuição da IC - outros & 23 & 71,1 & 40,4 \\
\hline Melhoria do processo de IC & 258 & 81,9 & 100,0 \\
\hline Melhoria do processo de IC - SIC & 28 & 8,9 & 10,9 \\
\hline Melhoria do processo de IC - evolução da IC & 19 & 6,0 & 7,4 \\
\hline Melhoria do processo de IC - reunião de informação & 13 & 4,1 & 5,0 \\
\hline Melhoria do processo de IC - monitoramento & 12 & 3,8 & 4,7 \\
\hline Melhoria do processo de IC - competências profissionais de IC & 11 & 3,5 & 4,3 \\
\hline Melhoria do processo de IC - ética & 11 & 3,5 & 4,3 \\
\hline Melhoria do processo de IC - análise de IC & 10 & 3,2 & 3,9 \\
\hline Melhoria do processo de IC - melhores práticas & 10 & 3,2 & 3,9 \\
\hline Melhoria do processo de IC - conceitual & 8 & 2,5 & 3,1 \\
\hline Melhoria do processo de IC - mensuração do valor de IC & 8 & 2,5 & 3,1 \\
\hline Melhoria do processo de IC - outros & 128 & 58,7 & 49,6 \\
\hline Total & 315 & 100,0 & - \\
\hline
\end{tabular}

Fonte: Elaborada pelos autores (2012-2013).

Nota: fi: frequência i; IC: Inteligência Competitiva; SIC: Sistema de Inteligência Competitiva.

Quadro 1. Estrutura científica e sistema de investigação da Inteligência Competitiva.

\begin{tabular}{|c|c|c|}
\hline Níveis de investigação & Produtos & Resultados \\
\hline Epistemológico & Paradigmas & $\begin{array}{l}\text { Cognitivo. } \\
\text { Físico. } \\
\text { Social. }\end{array}$ \\
\hline \multirow{8}{*}{ Científico } & Teorias & $\begin{array}{l}\text { Teoria do Conhecimento. } \\
\text { Teoria Geral de Administração. } \\
\text { Teoria da Decisão. } \\
\text { Teoria da Comunicação. } \\
\text { Teoria da Informação. } \\
\text { Teoria Geral dos Sistemas. } \\
\text { Teorias competitivas. } \\
\text { Teoria Geral da Inteligência. }\end{array}$ \\
\hline & Modelos & $\begin{array}{l}\text { Produção de Inteligência. } \\
\text { Contrainteligência. } \\
\text { Monitoramento Ambiental. } \\
\text { Sistema de Inteligência Competitiva. }\end{array}$ \\
\hline & & $\begin{array}{l}\text { Análise da indústria e da concorrência, principalmente os de Michael Porter. } \\
\text { Construção de cenários. } \\
\text { Benchmarking. }\end{array}$ \\
\hline & Métodos & Análise de redes sociais. \\
\hline & & Text mining. \\
\hline & & Early warning. \\
\hline & & Blindspot. \\
\hline & Técnicas & $\begin{array}{l}\text { Key Intelligence Topics. } \\
\text { Técnica de entrevista. }\end{array}$ \\
\hline Aplicado & Soluções de problemas & $\begin{array}{l}\text { Para melhoria do processo de Inteligência Competitiva. } \\
\text { Em outros processos organizacionais por meio da utilização da Inteligência Competitiva. }\end{array}$ \\
\hline
\end{tabular}

Fonte: Elaborado pelos autores (2014). 
sistêmica para a compreensão do objeto científico, nos níveis de investigação citados.

Apesar de as constatações iniciais apontarem para a carência de uma estrutura científica para a Inteligência Competitiva, os resultados da pesquisa mostraram que a mesma existia. Havia três paradigmas que regiam a pesquisa no campo da IC; os três formam o nível epistemológico e orientam a pesquisa no nível científico. Havia um corpo teórico que apoiava a pesquisa na área de IC e que era utilizado na solução de problemas de ordem aplicada. O mesmo era formado por teorias, leis, teoremas, modelos, métodos e técnicas que compõem o nível científico da disciplina. Havia contribuições práticas das pesquisas aplicadas no campo da IC relacionadas à melhoria do processo de Inteligência Competitiva e de contribuições da IC a outros processos organizacionais (nível aplicado), que retroalimentam o sistema.

O Quadro 1 apresenta uma síntese dessa estrutura científica ao mesmo tempo em que supre a falta de uma fundamentação teórica organizada e estruturada para a Inteligência Competitiva que contribua com a resolução de seus problemas de pesquisa. Complementando essa estrutura científica, destacou-se a diversidade de métodos de pesquisa utilizados pelos pesquisadores da área. Esses métodos perpassavam os diversos níveis de investigação, bem como várias áreas do conhecimento, caracterizando o sistema de investigação no campo da IC como interdisciplinar.

Concluiu-se também que o método e o modelo de pesquisa utilizados contribuíram para atingir o obje- tivo desta pesquisa, mostrando-se um instrumento para identificação e estruturação de novas disciplinas científicas. A principal limitação na aplicação do método foi o tempo necessário para avaliação dos documentos e para a identificação dos paradigmas que regiam a pesquisa na área, visto que, em alguns documentos, representavam uma avaliação subjetiva do pesquisador.

Foram diversas as contribuições do presente estudo. No campo científico: uma proposta de estrutura científica e sistema de investigação para a Inteligência Competitiva. No campo aplicado: (1) clareza do que é a atividade de IC e seus limites, de forma a contribuir com a estruturação dessa atividade nas organizações; (2) lista de métodos, modelos e técnicas que podem contribuir para a melhoria dos resultados da atividade de IC nas organizações; e (3) estrutura científica que contribui com a melhoria da capacitação do profissional de IC.

Destacam-se como possíveis desdobramentos desta pesquisa sua ampliação para a população identificada, bem como a inclusão de documentos científicos em outras línguas, como o francês, o alemão e o espanhol. Revisões periódicas ao longo do tempo também são pertinentes em função do avanço dos estudos na área.

\section{Colaboradores}

Todos os autores contribuíram na concepção e desenho do estudo, análise de dados e redação final.

\section{Referências}

Aguilar, F.J. Scanning the business environment. New York: Macmillan Company, 1967.

Capurro, R. Foundations of information science: Review and perspectives. In: International Conference on Conceptions of Library and Information Science, 1991, Finland. Proceedings electronics... Tampere: University of Tampere, 1991. Available from: <http://www.capurro.de/tampere91.htm>. Cited: Apr. $14,2004$.

Dishman, P.; Fleisher, C.; Knip, V. Chronological and categorized bibliography of key competitive intelligence scholarship: (1997 - present). Journal of Competitive Intelligence and Management, v.1, n.1, Pt.1, p.13-79, 2003.

Fleisher, C.S., Babette, B. Strategic and competitive analysis: Methods and approaches to analyzing business. New Jersey: Prentice Hall, 2003.
Fleisher, C.S.; Knip, V.; Dishman, P. Chronological and categorized bibliography of key competitive intelligence scholarship: (1990 - 1996). Journal of Competitive Intelligence and Management, v.1, n.2, Pt.2, p.11-86, 2003.

Fleisher, C.; Wright, S.; Tindale, R. Bibliography and assessment of key competitive intelligence scholarship: (2003-2006). Journal of Competitive Intelligence and Management, v.4, n.1, Pt.4, p.32-92, 2007.

Fuld, L.M. The new competitor intelligence: The complete resource for finding, analyzing, and using information about your competitors. New York: John Wiley \& Sons, 1995.

Gigch, J.P. van; Pipino, L.L. In search of a paradigm for the discipline of information systems. Future Computer Systems, v.1, n.1, p.71-97, 1986. 
Gil, A.C. Métodos e técnicas de pesquisa social. São Paulo: Atlas, 1991.

Kahaner, L. Competitive intelligence: How to gather, analyse, and use information to move your business to the top. New York: Simon \& Schuter, 1996.

Knip, V.; Dishman, P.; Fleisher, C. Chronological and categorized bibliography of key competitive intelligence scholarship: (The earliest writing - 1989). Journal of Competitive Intelligence and Management, v.1, n.3, Pt.3, p.10-79, 2003.

Kuhn, T.S. A estrutura das revoluções científicas. 9.ed. São Paulo: Perspectiva, 2006.

Marcial, E.C. Aspectos fundamentais da inteligência competitiva e a Ciência da Informação. 2013. Tese (Doutorado em Ciência da Informação) - Departamento de Ciência da Informação e Documentação, Universidade de Brasília, Brasília, 2013.

Marcial, E.C.; Ornelas, A.C. A Interdisciplinaridade da atividade de inteligência competitiva e o perfil dos doutores do Lattes vinculados a Inteligência Competitiva. In: Congresso Brasileiro de Biblioteconomia e Ciência da Informação, 22., 2007, Brasília. Anais eletrônicos... Brasília: FEBAB, 2007. 1 CD-ROM.

Marcial, E.C.; Suaiden, E.J. Inteligência competitiva: somente uma atividade prática ou também uma disciplina científica da ciência da informação? Tendências da Pesquisa Brasileira em Ciência da Informação, v.6, n.2, p.1-20, 2014.

Mendes, A.L.; Marcial, E.C., Fernandes, F. Fundamentos da inteligência competitiva. Brasília: Thesaurus, 2010. (Coleção Inteligência Competitiva, v.1).

Miller, J.P. Millennium intelligence: Understanding and conducting competitive intelligence in the digital age. New Jersey: CyberAge Books, 2000.
Mueller, S.P.M.; Miranda, A.; Suaiden, E.J. A pesquisa em Ciência da Informação no Brasil: análise dos trabalhos apresentados no IV Enancib, 2000. Revista de Biblioteconomia de Brasília, v.23/24, n.3, Esp., p.293-308, 1999/2000.

Platt, W. A produção de informação estratégica. Rio de Janeiro: Biblioteca do Exército Editora, 1974.

Popper, K.R. Conjecturas e refutações. 5.ed. Brasília: Editora UnB, 2008.

Porter, M.E. Vantagem competitiva: criando e sustentando um desempenho superior. 7.ed. Rio de Janeiro: Campus, 1992.

Prescott, J.E. The evolution of competitive intelligence. International Review of Strategic Management, v.6, n.1, p.71-90, 1995.

Prescott, J.E. Introduction to the special issue on the fundamentals of competitive intelligence. Competitive Intelligence Review, v.7, n.1, p.41-48, 1996. Edição Especial.

Prescott, J.E. The evolution of competitive intelligence: Designing a process for action. ProposalManagement, v.1, n.1, p.37-52, 1999.

Serpa, L.F. Epistemological assessment of current business intelligence archetypes. Competitive Intelligence Review, v.11, n.4, p.88-101, 2000.

Taborda, J.P.; Ferreira, M.D. Competitive intelligence: conceitos, práticas e benefícios. Cascais: Pergaminho, 2002.

Tarapanoff, K. Inteligência organizacional competitiva. Brasília: Editora UnB, 2001.

Tyson, K.W.M. The complete guide to competitive intelligence. Chicago: Division of Kirk Tyson Associates, 1998. 
